\title{
OPEN ILF2 enhances the DNA cytosine deaminase activity of tumor mutator APOBEC3B in multiple myeloma cells
}

\author{
Yasuhiro Kazuma ${ }^{1}$, Kotaro Shirakawa ${ }^{1 凶}$, Yusuke Tashiro $^{1}$, Hiroyuki Yamazaki ${ }^{1}$, \\ Ryosuke Nomura ${ }^{1}$, Yoshihito Horisawa ${ }^{1}$, Suguru Takeuchi ${ }^{1}$, Emani Stanford ${ }^{1}$, \\ Yoshinobu Konishi ${ }^{1}$, Hiroyuki Matsui ${ }^{1}$, Tadahiko Matsumoto ${ }^{1}$, Fumiko Tanabe ${ }^{2}$, \\ Ryo Morishita ${ }^{2}$, Shinji Ito ${ }^{3}$ \& Akifumi Takaori-Kondo ${ }^{1}$
}

DNA cytosine deaminase APOBEC3B (A3B) is an endogenous source of mutations in many human cancers, including multiple myeloma. A3B proteins form catalytically inactive high molecular mass (HMM) complexes in nuclei, however, the regulatory mechanisms of A3B deaminase activity in HMM complexes are still unclear. Here, we performed mass spectrometry analysis of A3B-interacting proteins from nuclear extracts of myeloma cell lines and identified 30 putative interacting proteins. These proteins are involved in RNA metabolism, including RNA binding, mRNA splicing, translation, and regulation of gene expression. Except for SAFB, these proteins interact with A3B in an RNAdependent manner. Most of these interacting proteins are detected in A3B HMM complexes by density gradient sedimentation assays. We focused on two interacting proteins, ILF2 and SAFB. We found that overexpressed ILF2 enhanced the deaminase activity of A3B by $30 \%$, while SAFB did not. Additionally, siRNA-mediated knockdown of ILF2 suppressed A3B deaminase activity by $30 \%$ in HEK293T cell lysates. Based on these findings, we conclude that ILF2 can interact with A3B and enhance its deaminase activity in HMM complexes.

\author{
Abbreviations \\ APOBEC3B Apolipoprotein B mRNA editing enzyme catalytic subunit-like protein 3B \\ co-IP Co-immunoprecipitation \\ siRNA Small interfering RNA \\ ssDNA Single-stranded DNA
}

Apolipoprotein B mRNA-editing enzyme catalytic polypeptide-like (APOBEC) family proteins have DNA cytidine deaminase activity that restricts retroviruses and retrotransposons by inducing hypermutations and degradation of replication intermediates. APOBECs are endogenous sources of DNA mutations that are often seen in many human cancers, including multiple myeloma ${ }^{1}$. Accumulation of APOBEC signature mutations is correlated with disease progression and poor overall survival in multiple myeloma ${ }^{2-5}$, especially in tumors with translocations between the IgH and $c-M A F(\mathrm{t}(14 ; 16))$ or MAFB $(\mathrm{t}(14 ; 20))$ genes. Among APOBEC3 enzymes, APOBEC3B (A3B) is mainly expressed in the nucleus ${ }^{6}$. We previously reported decreased deaminase activity and fewer APOBEC signature mutations upon shRNA-mediated A3B knockdown in myeloma cells, suggesting that, among APOBECs, A3B plays a major role in cytidine deamination-related mutagenesis in myeloma cells ${ }^{7}$.

Transcriptional and post-transcriptional mechanisms regulate A3B function. The canonical and non-canonical NF- $\kappa B$ pathway and b-Myb enhance A3B transcription, while E2F complexes suppress it ${ }^{8-11}$. Post-transcriptional regulatory mechanisms include protein kinase A mediated phosphorylation which inhibits A3B mutagenic activity $^{12}$ and alternative splicing of A3B which creates non-mutagenic isoforms ${ }^{13}$.

Recent studies have shown that co-factors of $\mathrm{A} 3 \mathrm{~B}$ can also affect its function. For example, the estrogen receptor (ER) recruits $\mathrm{A} 3 \mathrm{~B}$ at ER binding regions and introduces $\mathrm{C}$-to- $\mathrm{U}$ deamination which facilitates ER target gene

\footnotetext{
${ }^{1}$ Department of Hematology and Oncology, Graduate School of Medicine, Kyoto University, 54 Shogoin Kawahara-cho, Sakyo-ku, Kyoto 606-8507, Japan. ${ }^{2}$ CellFree Sciences Co., Ltd., Ehime, Japan. ${ }^{3}$ Medical Research Support Center, Graduate School of Medicine, Kyoto University, Kyoto, Japan. ${ }^{\circledR}$ email: kotash@kuhp.kyoto-u.ac.jp
} 
expression in breast cancer cells ${ }^{14}$. DHX9 interacts with A3B and inhibits its binding to pregenomic HBV RNA, attenuating the anti-HBV effect of $\mathrm{A}_{3} \mathrm{~B}^{15}$. An Epstein-Barr viral protein, BORF2, interacts with $\mathrm{A} 3 \mathrm{~B}$ through its catalytic domain and inhibits A3B deaminase activity ${ }^{16}$. Polycomb repressor complex 2 also interacts with A3B and reduces the occupancy of $\mathrm{H} 3 \mathrm{~K} 27 \mathrm{me} 3$ on promoters of the chemokine CCL2, modulating the microenvironment in hepatocellular carcinoma ${ }^{17}$.

APOBEC3 deaminase activity is strongly inhibited by RNA ${ }^{18}$. Another APOBEC3 protein, APOBEC3G (A3G), forms catalytically inactive high molecular mass (HMM) complexes and RNase A treatment disrupts HMM complexes into catalytically active low molecular mass (LMM) complexes ${ }^{19-22}$. A3B also forms HMM complexes, but RNase A treatment activates A3B without disrupting HMM into LMM complexes ${ }^{23}$. Multiple surface hydrophobic residues in its $\mathrm{N}$-terminal domain regulate the molecular assembly and deaminase activity of $\mathrm{A}_{3} \mathrm{~B}^{23}$. Mutation of these residues severely impairs interaction with multiple heterogeneous nuclear ribonucleoproteins (hnRNPs), which associate with A3B in an RNA-dependent manner ${ }^{23-25}$. These RNA-dependent interacting proteins seem to act as potential regulatory elements of $\mathrm{A} 3 \mathrm{~B}$ deaminase activity.

We hypothesized that the components of A3B HMM complexes may regulate A3B deaminase activity leading to APOBEC-mediated mutagenesis. In this study, we performed an interactome analysis using myeloma cell lines which express high levels of endogenous A3B. We previously developed myeloma cell lines which have a $3 \times$ FLAG-tag sequence inserted at the C-terminus of the $A 3 B$ gene via CRISPR/Cas9 editing, allowing us to directly analyze endogenous A3B expression using an anti-FLAG antibody ${ }^{26}$. Using these FLAG-knock-in cell lines, we showed that A3B HMM complexes are comprised of multiple RNA-binding proteins or hnRNPs. Among them, interleukin enhancer-binding factor 2 (ILF2) interacts with A3B in an RNA-dependent manner and enhances A3B deaminase activity.

\section{Results}

Proteomic analysis identifies APOBEC3B-interacting proteins in multiple myeloma cell lines. We immunoprecipitated (IP) endogenous FLAG-tagged A3B proteins from nuclear extracts of AMO1A3B-3 × FLAG-IRES-EGFP (AMO1-KI), RPMI8226-A3B-3 × FLAG-IRES-EGFP (RPMI-KI), and wild type cells (AMO1-WT and RPMI-WT) as negative controls ${ }^{26}$ with the anti-FLAG M2 antibody (Supplementary Fig. S1a, b), and analyzed the co-precipitated proteins by mass spectrometry (Supplementary Fig. S2). Mass spectrometry analyses successfully revealed peptides in the FLAG-IP samples (Supplementary Figs. S3, S4). Putative interacting proteins were selected as follows: unique peptide count ( $95 \%$ confidence) $\geqq 2$, normalized abundance $\geqq 2000$ and fold change relative to wild type cell lines $\geqq 2.0$ (analyzed with Progenesis QI). We identified 55 interacting protein candidates in AMO1 and 51 candidates in RPMI8226 cells, of which 30 candidates were common in both cell types (Fig. 1a,b; Supplementary Table S1; Supplementary dataset files 1 and 2).

A3B-interacting protein candidates are clustered largely into ribonucleoprotein complexes. To characterize the A3B-interacting protein candidates obtained by mass spectrometry, we employed the STRING interaction database (http://www.string-db.org/ ${ }^{27}$ ). We extracted the protein-protein interaction profiles of the 30 candidates that were common between AMO1 and RPMI8226 cells. Our analysis revealed that A3B-interacting protein candidates clustered largely into ribonucleoprotein complexes (Fig. 1). GO enrichment analysis demonstrated that a large proportion of them were associated with terms related to RNA metabolism, including RNA binding, mRNA splicing, translation, and regulation of gene expression (Supplementary dataset file 3). KEGG pathway analysis revealed that these interacting protein candidates belong to the spliceosome and ribosome complexes (Supplementary Fig. S1a,b).

Most of the interacting proteins bind to A3B in an RNA-dependent manner. We performed co-immunoprecipitation assays in myeloma cell lines to confirm the binding between A3B and the following representative A3B-interacting proteins: SAFB, SRSF7, Matrin-3, interleukin enhancer-binding factor 2 (ILF2), DHX9, RBMX, hnRNP A1, PTBP1, hnRNP K, hnRNP A3 and hnRNP C. Myeloma cell lysates were subjected to co-immunoprecipitation assays using anti-FLAG M2 affinity gel with or without RNase A. The anti-FLAG antibody specifically co-immunoprecipitated the interacting proteins that were detected by immunoblotting (Fig. 2 and Supplementary Fig. S6). We observed that in most cases the interaction was abolished in the presence of RNase A suggesting that the interaction was RNA-dependent. Among the 11 proteins tested in Fig. 2, SAFB was still detected after RNase A treatment, suggesting an RNA-independent interaction with A3B.

Most of the interacting proteins are components of HMM complexes. We also tested whether these proteins form HMM complexes with A3B in AMO1-KI and RPMI8226-KI cells by using density gradient sedimentation assays (Fig. 3, Supplementary Figs. S7, S8). We detected SAFB, SRSF7, Matrin-3, ILF2, DHX9, and RBMX in the high-density fractions, but not hnRNP A1, PTBP1, hnRNP K, hnRNP A3, or hnRNP C. After RNase A treatment, most of the putative interacting proteins were observed in the low-density fractions, but not in the high-density fractions. However, SAFB, SRSF7, and Matrin-3 remained in the high-density fractions even after RNase A treatment. Taken together, components of A3B HMM complexes are SAFB, SRSF7, and Matrin-3 via RNA-independent interactions, and ILF2, DHX9, and RBMX via RNA-dependent interactions.

We performed DNA deaminase activity assays with each fraction with or without RNase to evaluate the impact of these interactions on A3B functionality (Supplementary Fig. S9). Consistent with previous reports, RNase treatment increased deaminase activity. Interestingly, higher density fractions showed strongest activity after RNase treatment. It is possible that after RNA depletion, A3B and other DNA-binding proteins form RNAindependent high molecular masses. 


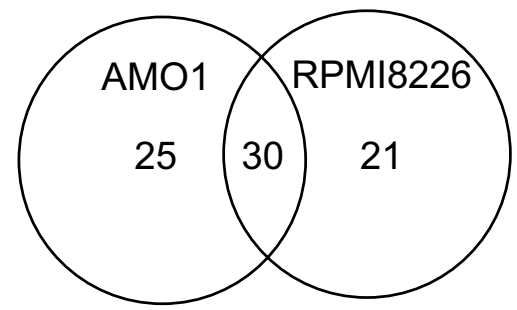

b

AM01

RPMI8226
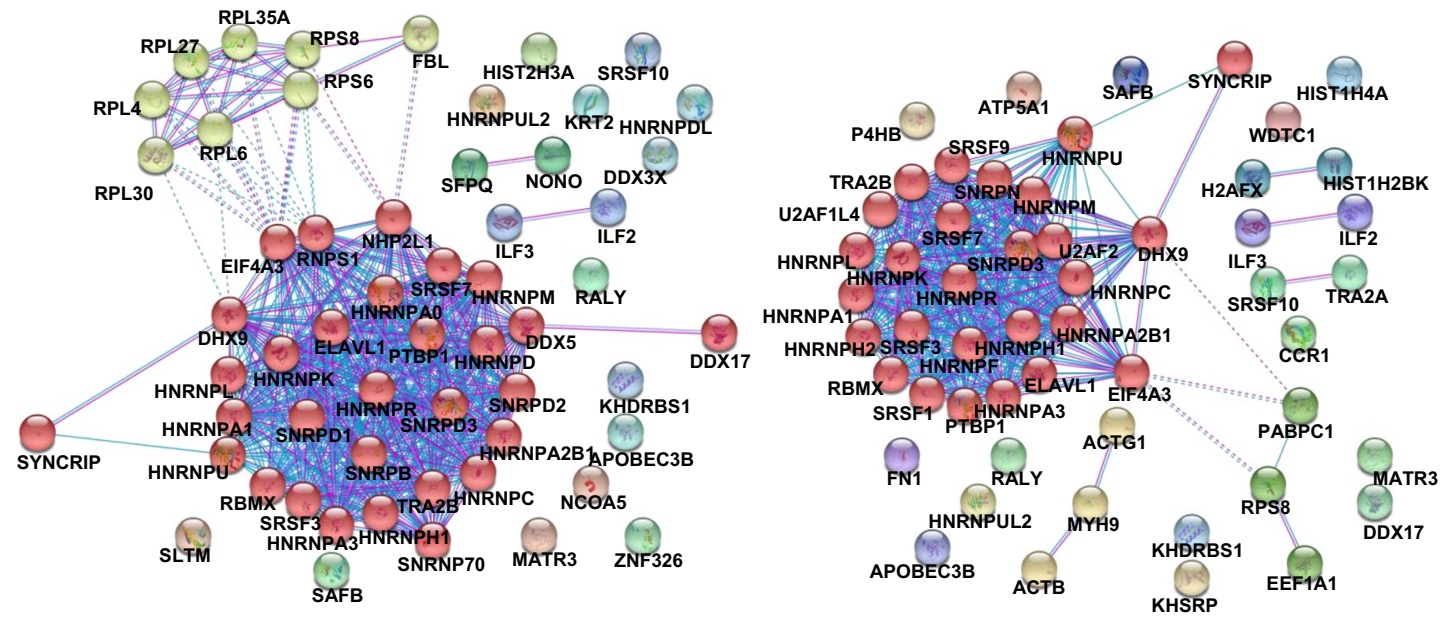

Figure 1. Analysis of the A3B-FLAG interactome in multiple myeloma cell lines. (a) Venn diagram of the proteins identified in MS analysis. We identified 55 interacting protein candidates in AMO1 and 51 candidates in RPMI8226 cells. As shown in Supplementary Table 1, 30 protein candidates were identified in both datasets. (b) Analysis based on the STRING interaction database showing the putative A3B-interacting proteins identified by MS clustered largely into ribonucleoprotein complexes (left, AMO1, right, RPMI8226).

\section{AM01}

input

IP: FLAG (A3B)

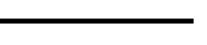

\section{WT KI}

RNase $\overline{-+}-+$

FLAG (A3B) 37-

SAFB

SRSF7

Matrin-3

ILF2

DHX9

IB

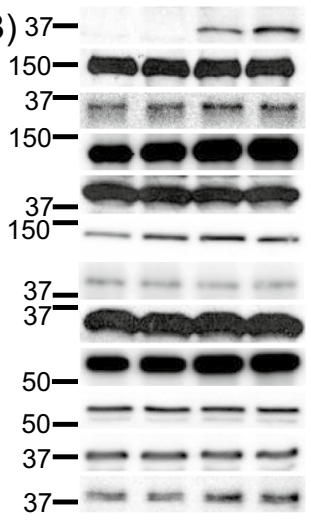

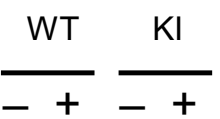

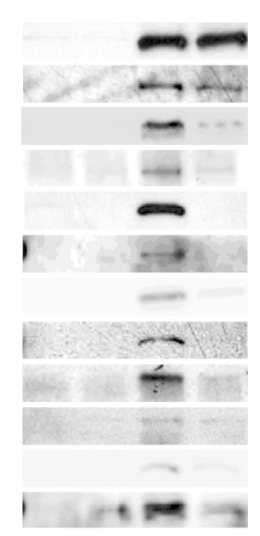

\section{RPMI8226}

input

IP: FLAG (A3B)
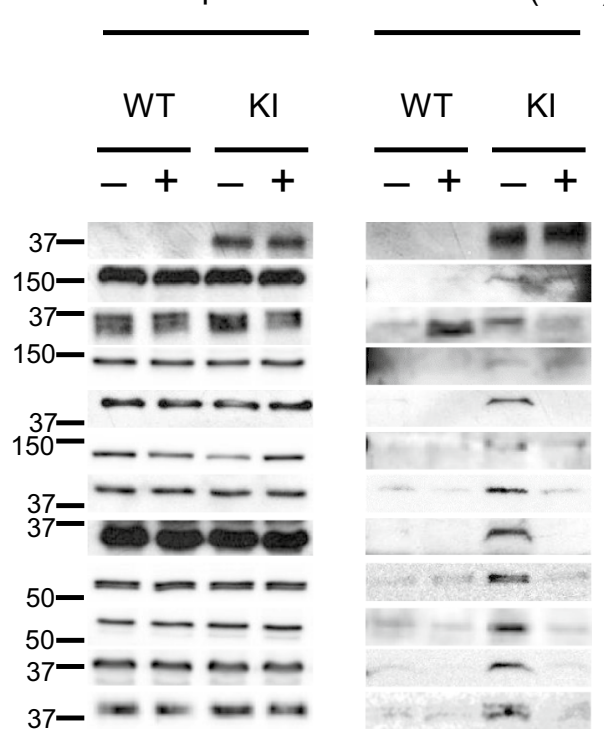
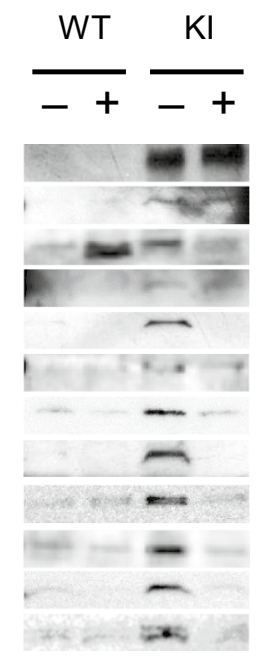

Figure 2. Validation of the interaction with $A 3 B$ by co immunoprecipitation. Immunoblot analysis of whole cell lysates (3\% input) prior to IP and after anti FLAG IP, with or without RNase A treatment (left, AMO1, right, RPMI8226). All images shown here are representative of three independent experiments. WT, wild type (parental) cell KI, $3 \times$ FLAG IRES EGFP knock in cell lines. Original blots are presented in Supplementary Figure S13. 


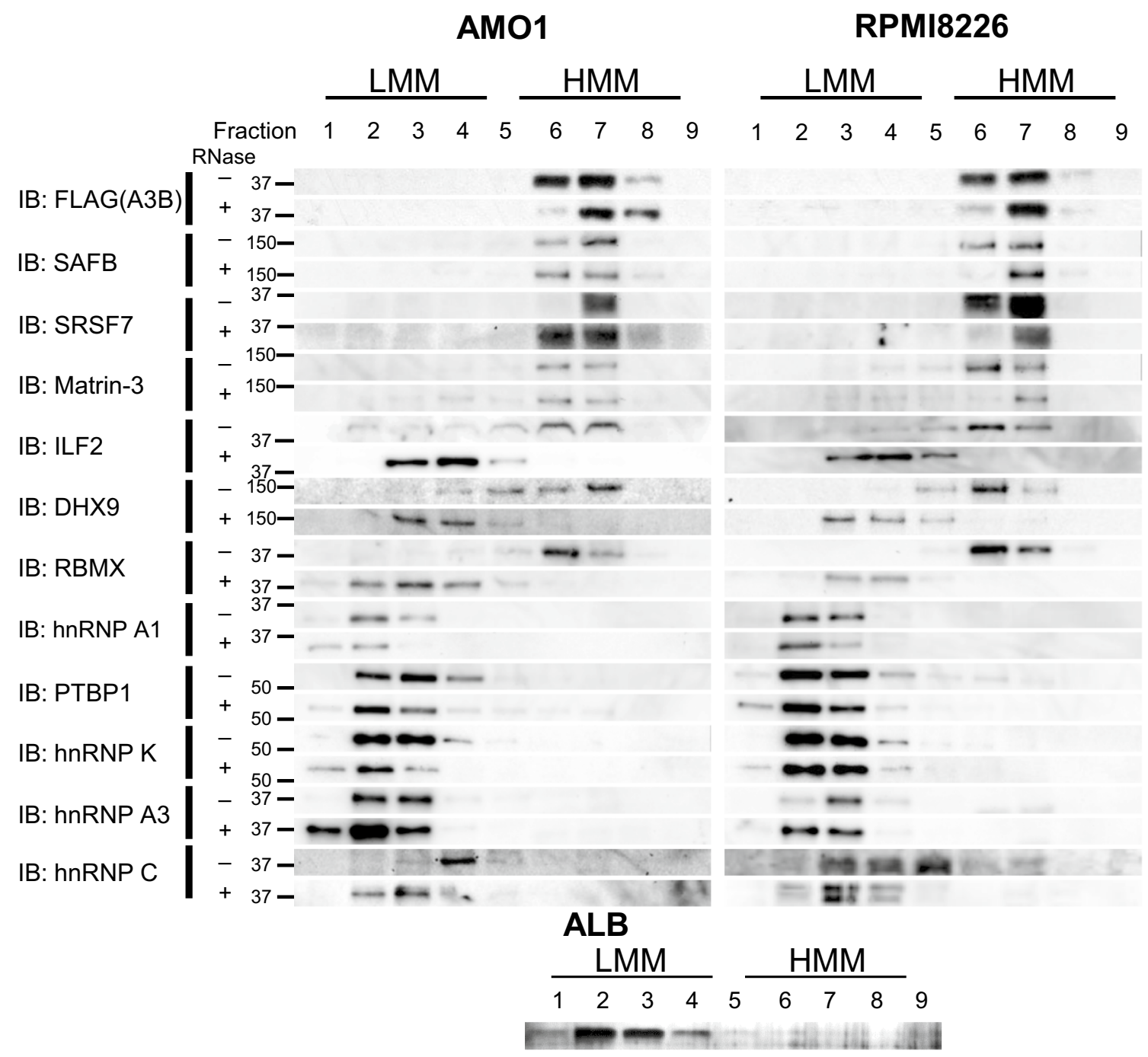

Figure 3. Density gradient sedimentation analysis showing that ILF2 is a component of HMM complexes. Whole cell lysates from AMO1 KI and RPMI8226 KI or lysis buffer containing albumin were loaded on to a discontinuous OptiPrep gradient (436\%) and centrifuged at 25,000 rpm for $14 \mathrm{~h}$ at $4{ }^{\circ} \mathrm{C}$. The separated fractions were analysed by Western blotting with specific antibodies against FLAG and each interactor candidate (left, AMO1, right, RPMI8226). The position of albumin as a molecular weight marker was confirmed using silver staining. LMM Low molecular mass. HMM High molecular mass. Original blots are presented in Supplementary Figure S14.

ILF2 co-localizes with $A 3 B$ in the nucleus and enhances the cytidine deaminase activity of A3B. We further characterized two A3B interacting proteins: $S A F B$, which is a nuclear matrix protein known to form molecular assemblies ${ }^{28}$, and which interacted with A3B in an RNA-independent manner (shown in Fig. 2), and ILF2, which interacted with A3B in an RNA-dependent manner and which is the only protein shown to contribute to multiple myeloma pathogenesis ${ }^{29}$ among the interacting proteins listed so far.

First, we checked the intracellular localization of these proteins in AMO1-KI cells by immunofluorescence microscopy. We observed that both SAFB and ILF2 localized in the nucleus. Taking into consideration also the results of co-IP and density gradient sedimentation analyses, these proteins co-localize with A3B in the nucleus (Fig. 4a,b). We confirmed the interactions in the nucleus using co-IP and reciprocal co-IP with nuclear lysates from HEK293T cells (Supplementary Fig. S10a,b).

Second, to examine whether these interacting proteins affect A3B function, we measured the deaminase activity of A3B in vitro using purified proteins produced in a wheat germ cell-free expression system (Supplementary Fig. S11a-d). A3B-C terminal domain (CTD), ILF2 and oligonucleotides were mixed and rotated for $30 \mathrm{~min}$ at $37^{\circ} \mathrm{C}$. We used the fluorescent protein Venus as a negative control because it doesn't interact with A3B. ILF2 enhanced A3B deaminase activity compared to Venus in a dose-dependent manner (Supplementary Fig. S12a,b). We could not test SAFB in this assay because we were not able to purify in vitro-translated SAFB proteins by a standard method.

Third, we measured the deaminase activity of A3B using HEK293T cell lysates with overexpressed A3B and interacting proteins as previously described ${ }^{12}$. Overexpression of ILF2 enhanced the deaminase activity of A3B 


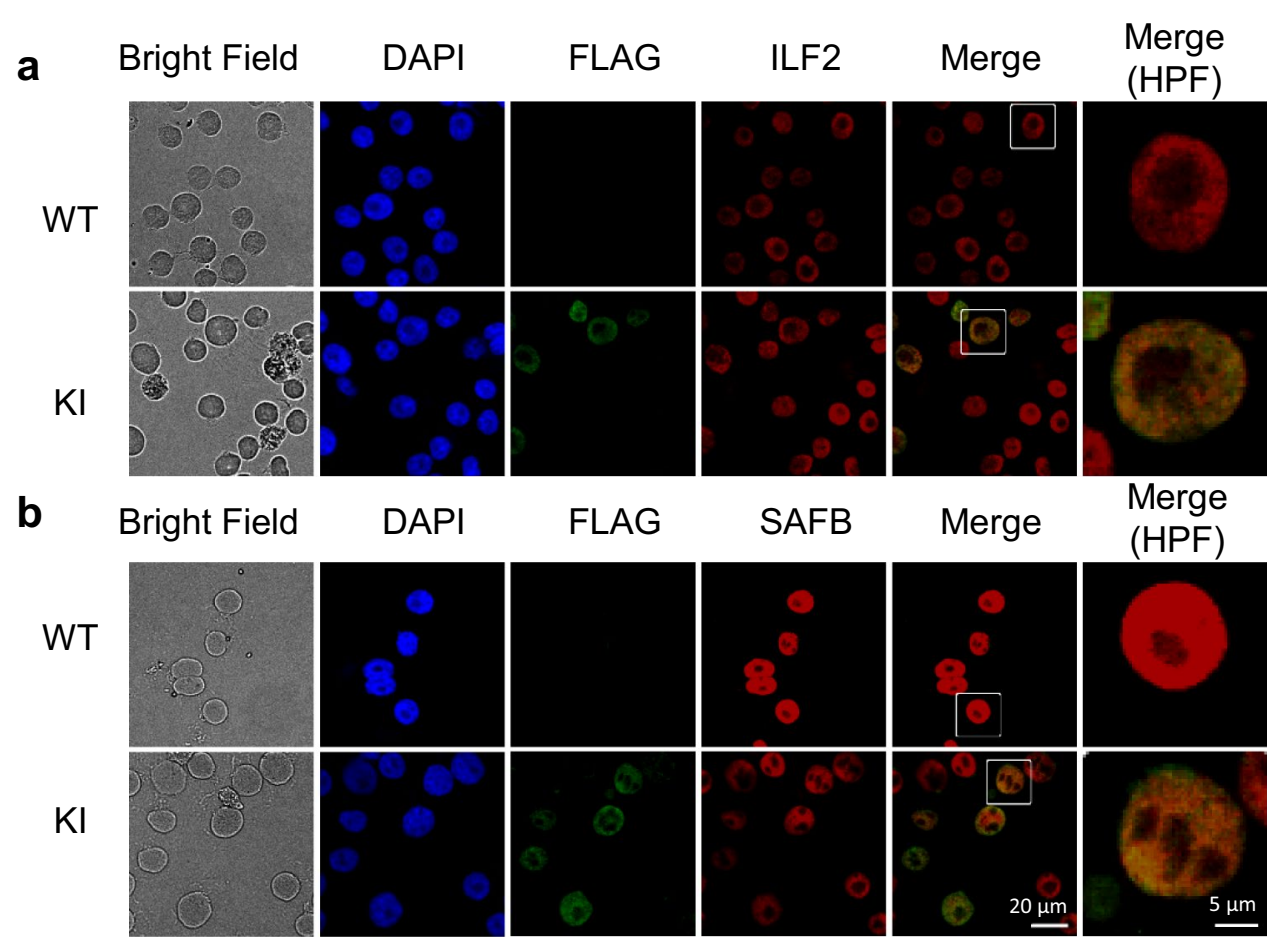

Figure 4. A3B co localizes with ILF2 and SAFB in the nucleus. AMO1 KI cells were subjected to immunofluorescence using Alexa Fluor 555 labelled anti mouse secondary antibody. Endogenous ILF2 (a) and SAFB (b) were visualized using Alexa Fluor 647 labelled anti rabbit secondary antibody and DAPI was used to stain the nucleus. Image acquisition was performed with a confocal laser scanning microscope (BZ X800, KEYENCE). WT, Wild type (parental) cell lines. KI, $3 \times$ FLAG IRES EGFP knock in cell lines. HPF High power field.

by $30 \%$, but of SAFB did not (Fig. 5a,b). Additionally, siRNA-mediated knockdown of ILF2 suppressed A3B deaminase activity by $30 \%$ (Fig. $5 \mathrm{c}$,d). Taken together, these data indicate that ILF2 positively regulates the deaminase activity of $\mathrm{A} 3 \mathrm{~B}$.

\section{Discussion}

RNA-mediated formation of HMM ribonucleoprotein complexes plays a crucial role in APOBEC deaminase activity. Mishra et al. reported that mass spectrometry-based proteomics using HEK293T cells with overexpressed A3B identified A3B-interacting proteins, however, the precise role of these proteins has not been elucidated ${ }^{24}$. In this study, we have performed for the first time a proteomic analysis of the A3B interactome in a multiple myeloma context. We used myeloma cells that endogenously express A3B and identified 30 putative interacting proteins. Most of the putative interacting proteins were RNA-binding proteins including hnRNPs, ribosomal proteins and splicing factors. Co-IP experiments showed that, except for SAFB, the interactions of these proteins with A3B were RNA-dependent. We also showed that these interacting proteins exist in HMM complexes and most of them move to low-molecular fractions when treated with RNase A. Additionally, immunofluorescence microscopy studies showed that ILF2 and SAFB co-localize with A3B in the nucleus. Lastly, functional assays showed that ILF2 enhanced the deaminase activity of A3B.

Our results indicate that the components of A3B HMM complexes may play important roles in regulating its deaminase activity. In terms of other APOBEC family proteins, components of the complexes they are part of have been reported to modulate their catalytic activity. For example, activation-induced cytidine deaminase (AID) monomers interact with hnRNP K, whereas AID dimers interact with hnRNP $\mathrm{L}^{30}$. hnRNP K is involved in DNA cleavage events associated with somatic hypermutation, while hnRNP L is involved in DNA recombination events associated with class switch recombination ${ }^{31}$, suggesting that different interacting proteins play different roles in the function of APOBEC-protein complexes. However, little is known about how protein-protein interactions affect the deaminase activity of APOBEC-protein complexes. Four tyrosine mutations and W127A in the N-terminal domain of A3B impair HMM assembly and A3B deaminase activity ${ }^{23}$. Other components of the HMM complexes also bind to ssDNA and might provide substrates to adjacent A3B, facilitating deamination. RNase A treatment of the complexes drastically elevates deaminase activity ${ }^{18,23}$ although it disrupts RNAdependent interaction with activating co-factors including ILF2. Additionally, our density sedimentation analysis showed that the fraction with the highest deaminase activity shifted to higher molecular weight fractions after RNase treatment. It is possible that RNA-mediated A3B inhibition might be more important than co-factor mediated activation. Another possibility is that A3B HMM may be converted into RNase-resistant complexes with unknown activating co-factors. 
a

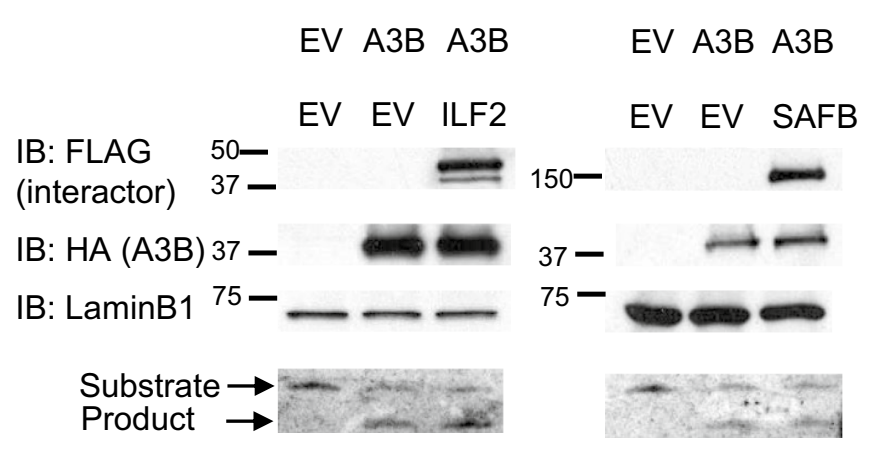

b

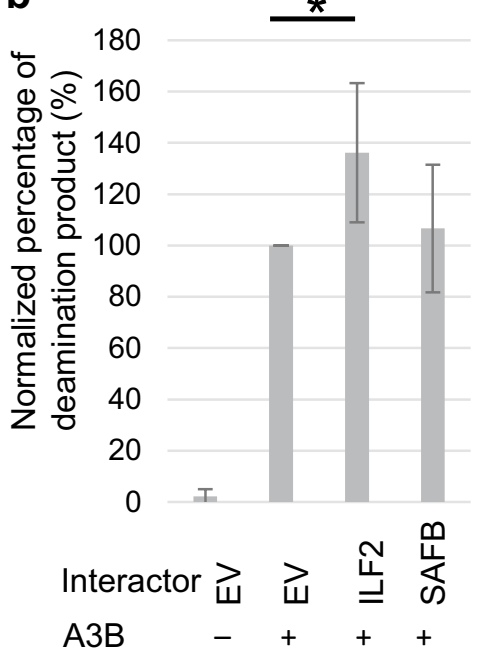

IB: ILF2

C $\begin{array}{lll}\text { EV } & \text { A3B } & \text { A3B } \\ \text { NC } & \text { NC } & \text { si ILF2 }\end{array}$
EV A3B A3B NC NC si SAFB
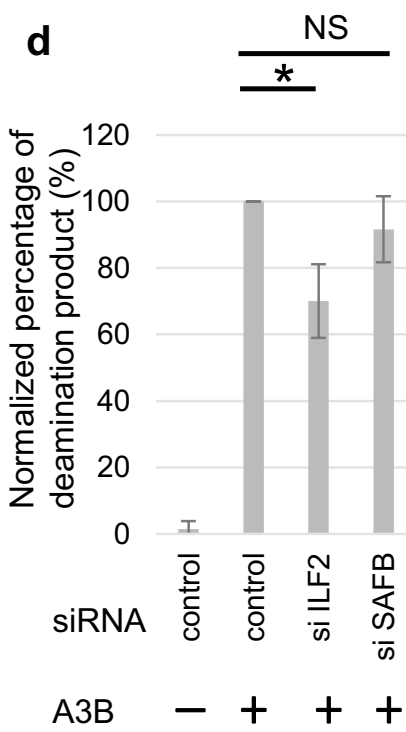

Figure 5. ILF2 enhances A3B cytidine deaminase activity. (a) Immunoblot analysis of interactor overexpressing HEK293T cell lysate (upper panel) and representative TBE urea PAGE analysis of A3B cytidine deaminase activity (lower panel). LaminB1 is an endogenous control. (b) Quantification of the cytidine deaminase activity data in five biologically independent experiments. Deaminated/total (deaminated and aminated) percentage of product was calculated and normalized by the control sample overexpressing A3B without interactors. (c) Immunoblot analysis of interactor knocked down HEK293T cell lysate and representative TBE urea PAGE analysis of A3B cytidine deaminase activity. (d) Quantification of the cytidine deaminase activity data in five biologically independent experiments. Deaminated/total (deaminated and aminated) percentage of product was calculated and normalized by the sample overexpressing A3B with control siRNA. Values are means standard deviations (error bar). Asterisks ${ }^{*}$ ) show statistically significant difference $p<0.05$ ). EV Empty vector, NC Negative control (scramble siRNA). Original blots and gels are presented in Supplementary Figure S15.

ILF2 is overexpressed in various tumor types, including multiple myeloma ${ }^{29}$. Of note, ILF2 is located in the chromosomal region 1q21. 1q21 amplification occurs in approximately 30 to $50 \%$ of newly diagnosed and 50 to $80 \%$ of relapsed/refractory multiple myeloma cases, and is associated with disease progression and drug resistance ${ }^{32-36}$. The candidate genes which participate in the pathogenicity of $1 \mathrm{q} 21$ amplification include $M U C 1^{37}$, $M C L 1^{38}, P D Z K 1^{39}, I L 6 R^{40}, B C L 9^{41}, C K S 1 B^{42}, P S M D 4^{43}$ and $I L F 2^{29}$, but the crucial driver oncogenes have not been identified yet. ILF2 induces drug resistance in myeloma cells via mRNA processing and the stabilization of transcripts involved in homologous recombination in response to DNA damage ${ }^{29}$. In this study, we found that ILF2 interacts with A3B and enhances A3B deaminase activity, suggesting that ILF2 may contribute to clonal evolution or drug resistance not only by enhancing mRNA splicing of DNA damage response proteins, but also by increasing A3B deaminase activity. 
In summary, we demonstrate that, in myeloma cells, A3B HMM complexes are comprised of multiple RNAbinding proteins and some of them can affect A3B deaminase activity. However, it remains to be established whether these interacting proteins regulate $\mathrm{A} 3 \mathrm{~B}$ deaminase activity in myeloma cells in vivo. It is possible that other co-factors interact with A3B and play a different role in certain conditions such as under replication stress or after viral infection. Further investigation is required to elucidate which interactions are meaningful in physiological settings.

\section{Methods}

Cell lines and cell culture. Two human myeloma cell lines, AMO1 and RPMI8226 cells were maintained in RPMI1640 (Nacalai Tesque) containing 20\% FBS and 1\% PSG (Invitrogen). HEK293T cells were maintained in DMEM (Nacalai Tesque) containing 10\% FBS and 1\% PSG. A3B reporter cell lines that contain a $3 \times$ FLAG tag at the C-terminus of the $A 3 B$ gene: AMO1-KI and RPMI8226-KI cells were generated as previously reported ${ }^{26}$.

Co-immunoprecipitation assays for mass spectrometry analysis. For co-immunoprecipitation with an anti-FLAG antibody for mass spectrometry analysis, nuclear extracts were prepared as previously described $^{44} .5 \times 10^{7}$ cells were briefly washed once with phosphate-buffered saline and the cell pellet were suspended in Hypobuffer (10 mM HEPES-KOH pH 7.9, $10 \mathrm{mM} \mathrm{KCl}$ ). After incubating for 15 min on ice, TritonX-100 was added. The lysed cellular suspension was briefly vortexed and microcentrifuged for 30 min at $4{ }^{\circ} \mathrm{C}$. The supernatant was discarded as cytoplasmic extract. The pellet was resuspended in high salt radioimmunoprecipitation (RIPA) buffer (50 mM Tris- $\mathrm{HCl}$ pH 8.0, $450 \mathrm{mM} \mathrm{NaCl}, 1 \%$ (v/v) Triton X-100, 0.1\% (v/v) SDS, $0.1 \%(\mathrm{v} / \mathrm{v})$ sodium deoxycholate) and agitated for $30 \mathrm{~min}$ at $4^{\circ} \mathrm{C}$. After centrifugation, the supernatant was mixed with 2 volumes of no salt RIPA buffer (50 mM Tris- $\mathrm{HCl} \mathrm{pH} \mathrm{8.0,1 \%} \mathrm{(v/v)} \mathrm{Triton} \mathrm{X-100,} \mathrm{0.1 \%} \mathrm{(v/v)} \mathrm{SDS,}$ $0.1 \%(\mathrm{v} / \mathrm{v})$ sodium deoxycholate, $5 \%$ Glycerol). After pre-clearing with $10 \mu \mathrm{L}$ of FG beads (Tamagawa Seiki), the supernatant was incubated with antibody-coupled FG beads for $2 \mathrm{~h}$ at $4^{\circ} \mathrm{C}$. FG beads were conjugated with the anti-FLAG antibody according to the manufacturer's instructions and then used for immunoprecipitation. The beads were washed five times with low salt RIPA buffer $(50 \mathrm{mM}$ Tris- $\mathrm{HCl} \mathrm{pH} \mathrm{8.0,150} \mathrm{mM} \mathrm{NaCl,} \%$ (v/v) Triton X-100, 0.1\% (v/v) SDS, $0.1 \%(\mathrm{v} / \mathrm{v})$ sodium deoxycholate) and then incubated for 30 min after adding FLAG elution buffer (TBS supplemented with $150 \mu \mathrm{g} / \mathrm{mL}$ of $3 \times$ FLAG peptide). The protease inhibitor cocktails (Nacalai Tesque) and PhosSTOP (Roche) were added to Hypobuffer and RIPA buffer just before use. The eluted samples were analyzed by silver staining using the Silver Staining MS kit (Wako, Osaka, Japan) according to the manufacturer's instructions.

Mass spectrometry. The eluted proteins were precipitated with cold acetone, dried and resuspended in $8 \mathrm{M}$ urea/30 mM ammonium bicarbonate. After reduction and alkylation with dithiothreitol and iodoacetamide, the proteins were digested with trypsin overnight, then purified using Pierce C18 spin columns (Thermo Fisher Scientific), dried and resuspended in $0.1 \%$ formic acid. The separation was carried out using Nano-LCUltra 2D-plus equipped with cHiPLC Nanoflex (Eksigent, Dublin, CA, USA) in trap-and-elute mode, with trap column $(200 \mu \mathrm{m} \times 0.5 \mathrm{~mm}$ ChromXP C18-CL $3 \mu \mathrm{m} 120 \AA$ (Eksigent) $)$ and analytical column $(75 \mu \mathrm{m} \times 15 \mathrm{~cm}$ ChromXP C18-CL $3 \mu \mathrm{m} 120 \AA$ (Eksigent)). The binary gradients used for the separation were as follows: A98\%/ $\mathrm{B} 2 \%$ to $\mathrm{A} 66.8 \% / \mathrm{B} 33.2 \%$ in $125 \mathrm{~min}, \mathrm{~A} 66.8 \% / \mathrm{B} 33.2 \%$ to $\mathrm{A} 2 \% / \mathrm{B} 98 \%$ in $2 \mathrm{~min}, \mathrm{~A} 2 \% / \mathrm{B} 98 \%$ for $5 \mathrm{~min}, \mathrm{~A} 2 \% / \mathrm{B} 98 \%$ to $\mathrm{A} 98 \% / \mathrm{B} 2 \%$ in $0.1 \mathrm{~min}$, and $\mathrm{A} 98 \% / \mathrm{B} 2 \%$ for $17.9 \mathrm{~min}$, in which $0.1 \%$ formic acid/water and $0.1 \%$ formic acid/ acetonitrile were used as solvents $\mathrm{A}$ and $\mathrm{B}$ respectively. The flow rate was $300 \mathrm{~nL} / \mathrm{min}$. The analytical column temperature was set to $40^{\circ} \mathrm{C}$. The eluates were infused on-line to a mass spectrometer (TripleTOF $5600+$ System with NanoSpray III source and heated interface (SCIEX, Framingham, MA, USA)) and ionized in an electrospray ionization-positive mode. Data acquisition was carried out with an information-dependent acquisition method.

Label-free quantification of the relative protein abundance. The acquired datasets were analyzed using ProteinPilot 5.0.1 (SCIEX) with the UniProtKB/Swiss-Prot human database (May 2018) appended with known common contaminants (SCIEX). The quality of the database search was confirmed by the false discovery rate analysis in which the reversed amino acid sequences were used as decoy. The reliability of protein identifications was evaluated by the number of identified peptides with confidence of at least 95\%, and Unused ProtScores that were calculated by the Pro Group algorithm (SCIEX). Relative abundances of the identified proteins were estimated on the platform of Progenesis QI for Proteomics 4.1 (Nonlinear Dynamics, Newcastle upon Tyne, UK). All raw data files in wiff format (SCIEX) were imported to generate aggregates, and the peptide identification results by ProteinPilot with confidence of at least $95 \%$ were used for assignment. Label-free quantification of proteins was performed by relative quantitation using the Hi-N method (Nonlinear Dynamics).

Functional classification and network of interactions. Protein-protein interaction networks were constructed with the STRING database ${ }^{27}$. GO annotation and KEGG pathway enrichment analysis was conducted using DAVID Functional Annotation Tool ${ }^{45-49}$.

Antibodies. Antibodies used for protein analysis were as follows: Anti-FLAG M2 antibody (F3165 SigmaAldrich), Anti-ILF2 antibody (ab113205 Abcam), Anti-SAFB antibody [EPR13588] (ab187650 Abcam), AntiRNA Helicase A antibody (ab26271 Abcam), Anti-HNRPA3/HNRNPA3 antibody (ab78300 Abcam), AntiMatrin-3 antibody [EPR10635(B)] (ab151714 Abcam), Anti-SFRS7/SRSF7 antibody (ab138022 Abcam), hnRNP C1/C2 (D6S3N) Rabbit mAb (\#91,327 Cell Signaling Technology), RBMX/hnRNP G (D7C2V Cell Signaling 
Technology) Rabbit mAb (\#14,794 Cell Signaling Technology), PTBP1 (E4I3Q) Rabbit mAb (\#57,246 Cell Signaling Technology), hnRNP K (R332) Antibody (\#4675 Cell Signaling Technology), Anti-hnRNP A1 Antibody (4B10) (sc-32301 Santa Cruz Biotechnology), Anti-Histone H3 Antibody, CT, pan (07-690 Sigma-Aldrich), Anti-Lamin B1 antibody [EPR8985(B)] (ab133741 Abcam). The primary antibodies were detected using Rabbit IgG HRP Linked Whole Ab (NA934 Cytiva) or Mouse IgG HRP Linked Whole Ab (NA931 Cytiva). The bands were visualized using Pierce ECL Plus Western Blotting Substrate (Thermo Fisher Scientific).

Co-immunoprecipitation assays for immunoblotting. Myeloma cells were lysed with low salt RIPA buffer containing complete Protease Inhibitor Cocktail (Roche) and PhosSTOP (Roche). The lysates were immunoprecipitated using the ANTI-FLAG M2 Affinity Gel (A2220 Sigma-Aldrich) at $4{ }^{\circ} \mathrm{C}$ for $2 \mathrm{~h}$ in the presence or absence of RNase A, DNase-free, followed by immunoblotting.

HEK293T cells were transfected with expression vectors for A3B-FLAG using the XtremeGENE HP DNA Transfection Reagent. The nuclear extract was collected as described for the sample preparation for mass spectrometry and immunoprecipitated using the anti-FLAG, anti-ILF2 or anti-SAFB antibody along with Protein G beads at $4{ }^{\circ} \mathrm{C}$, followed by immunoblotting.

Density gradient sedimentation. Nine layers of 4 to $36 \%$ sucrose were prepared in GST lysis buffer (150 mM NaCl, $25 \mathrm{mM}$ HEPES pH 7.4, 0.5\% Triton-X100, $1 \mathrm{mM} \mathrm{MgCl}_{2}, 1 \mathrm{mM} \mathrm{ZnCl}_{2}, 10 \%$ Glycerol). The whole cell extract, which was made with GST lysis buffer containing protease inhibitor cocktail (Roche), was loaded on top of the gradient and ultracentrifuged for $14 \mathrm{~h}$ at 25,000 rpm (CP65; Hitachi Koki) in a SW41Ti rotor. After ultracentrifugation, 10 fractions were collected from the top of the gradient and subjected to immunoblotting and gel-based cytidine deaminase activity assay.

Immunofluorescence assays. Myeloma cells were air-dried and fixed in $3.7 \%$ formaldehyde in phosphate-buffered saline (PBS) for $10 \mathrm{~min}$ at room temperature on glass slides using Shandon cytospin 2 (Thermo Fisher Scientific). Fixed cells were permeabilized, reduced and denatured for $30 \mathrm{~min}$ at room temperature in PBS buffer containing $0.5 \%$ SDS, $5 \% \beta$-mercaptoethanol and 10\% FBS. Then, cells were washed three times with PBS containing $4 \%$ FBS and $0.1 \%$ Triton X-100 (PET buffer) and incubated with the anti-FLAG antibody (1:200 dilution) and either rabbit anti-ILF2 (1:200 dilution), or anti-SAFB (1:200 dilution) antibody for $1 \mathrm{~h}$. Cells were then washed three times with PET buffer and incubated with Alexa Fluor 555 and Alexa Fluor 647 labeled secondary antibodies (A11078; A11037 Cell Signaling Technology) for $1 \mathrm{~h}$ in the dark. All antibodies were diluted with 3\% BSA and $0.5 \%$ Tween in PBS. Slides were mounted in VECTASHIELD with DAPI (Vector Labs) and observed with a confocal laser scanning microscope (BZ-X800, KEYENCE).

Knockdown experiments. Transfection and co-transfection were carried out using Lipofectamine RNAiMAX (Invitrogen) according to the manufacturer's instructions. Cells were harvested $72 \mathrm{~h}$ after transfection. Short interfering RNA (siRNA) duplexes in this study were purchased from Thermo Fisher Scientific (ILF2: Silencer select pre-Designed siRNA s7400, s7399; SAFB: Silencer select pre-Designed siRNA s12452, s12453; non-target control: Silencer Select Negative Control \#1 siRNA $(4,390,843))$.

Protein expression and purification using a wheat germ cell-free expression system. Histagged A3B (C-terminal domain) was expressed in a wheat germ cell-free expression system using the WEPRO7240H Expression Kit (CellFree Sciences, Matsuyama, Japan). Brij-35 at a final concentration of 0.04\% and zinc acetate at a final concentration of $1 \mu \mathrm{M}$ were added to the translation reaction solution. Expressed proteins were purified by affinity chromatography with nickel-Sepharose.

Flag/GST-tagged ILF2, hnRNP A1, and Venus were expressed in a wheat germ cell-free expression system using the WEPRO7240G Expression Kit (CellFree Sciences, Matsuyama, Japan). Brij-35 at a final concentration of $0.04 \%$ was added to the translation reaction solution. Expressed proteins were purified by affinity chromatography with glutathione-Sepharose.

Gel-based cytidine deaminase activity assays. To prepare cell lysates with overexpressed A3B and interacting proteins, HEK293T cells were transfected with expression vectors for A3B and for each interactor using XtremeGENE HP DNA Transfection Reagent (Roche). The cells were harvested $48 \mathrm{~h}$ later, lysed with $120 \mu \mathrm{l}$ of GST lysis buffer/well and used for gel-based cytidine deaminase activity (CDA) assays. To prepare interactor-depleted lysates, HEK293T cells $\left(5 \times 10^{5}\right)$ were transfected with $60 \mathrm{nM}$ interactor-specific or scrambled siRNA. At $24 \mathrm{~h}$, the cells were transfected with expression vectors for A3B. At $72 \mathrm{~h}$, the cells were harvested, lysed and assayed. $5 \mu \mathrm{l}$ of HEK293T cell lysate and 1 pmol of single-stranded DNA oligonucleotide (ATTATT ATTATTCAAATGGATTTATTTATTTATTTATTTATTT) with 5'-attached FAM were incubated with 0.005 units of UDG and $3.75 \mu \mathrm{l}$ of reaction buffer in $10 \mu \mathrm{l}$ reaction volume for $2 \mathrm{~h}$ at $37^{\circ} \mathrm{C}$. Subsequently, the oligo products were incubated in $100 \mathrm{mM} \mathrm{NaOH}$ for $30 \mathrm{~min}$ at $37^{\circ} \mathrm{C}$, stained with loading dye, and denatured, followed by electrophoresis in $20 \%$ Tris/urea-acrylamide gel, which was then visualized with Image Quant LAS 500. Purified A3B-CTD, ILF2, and Venus were produced using the wheat germ cell-free expression system ${ }^{50,51}$ and were provided by Cell Free Sciences (Supplementary Fig. S11a-d). For the gel-based CDA assays using purified proteins, we used purified A3B-CTD instead of lysate. Before the reaction, 1 pmol of A3B-CTD and 2, 4, or $8 \mathrm{pmol}$ of each interactor were mixed and rotated for $30 \mathrm{~min}$ at $37^{\circ} \mathrm{C}$. For the assays using the gradient fractions from myeloma cell lines, each lysate fraction, DNA substrates and UDG were mixed and incubated for $6 \mathrm{~h}$ at $37^{\circ} \mathrm{C}$ before the assay. 
Statistical analysis. The signal intensity of the bands in Western blot membranes or Urea-PAGE gels used in CDA assays was quantified using ImageJ version $1.51 \mathrm{~d}$ (https://imagej.nih.gov/ij/). P values were calculated by paired $\mathrm{T}$ test, and $p<0.05$ was defined as significant difference.

Received: 27 July 2021; Accepted: 19 January 2022

Published online: 10 February 2022

\section{References}

1. Burns, M. B. et al. APOBEC3B is an enzymatic source of mutation in breast cancer. Nature 494, 366-370. https://doi.org/10.1038/ nature11881 (2013).

2. Walker, B. A. et al. Identification of novel mutational drivers reveals oncogene dependencies in multiple myeloma. Blood 132, 587-597. https://doi.org/10.1182/blood-2018-03-840132 (2018).

3. Maura, F. et al. Biological and prognostic impact of APOBEC-induced mutations in the spectrum of plasma cell dyscrasias and multiple myeloma cell lines. Leukemia 32, 1044-1048. https://doi.org/10.1038/leu.2017.345 (2018).

4. Bolli, N. et al. Genomic patterns of progression in smoldering multiple myeloma. Nat. Commun. 9, 3363. https://doi.org/10.1038/ s41467-018-05058-y (2018).

5. Walker, B. A. et al. APOBEC family mutational signatures are associated with poor prognosis translocations in multiple myeloma. Nat. Commun. 6, 6997. https://doi.org/10.1038/ncomms7997 (2015).

6. Lackey, L. et al. APOBEC3B and AID have similar nuclear import mechanisms. J. Mol. Biol. 419, 301-314. https://doi.org/10. 1016/j.jmb.2012.03.011 (2012).

7. Yamazaki, H. et al. Endogenous APOBEC3B overexpression constitutively generates DNA substitutions and deletions in myeloma cells. Sci. Rep. 9, 7122. https://doi.org/10.1038/s41598-019-43575-y (2019).

8. Roelofs, P. A. et al. Characterization of the mechanism by which the RB/E2F pathway controls expression of the cancer genomic DNA deaminase APOBEC3B. Elife https://doi.org/10.7554/eLife.61287 (2020).

9. Maruyama, W. et al. Classical NF-kappaB pathway is responsible for APOBEC3B expression in cancer cells. Biochem. Biophys. Res. Commun. 478, 1466-1471. https://doi.org/10.1016/j.bbrc.2016.08.148 (2016).

10. Leonard, B. et al. The PKC/NF-kappaB signaling pathway induces APOBEC3B expression in multiple human cancers. Can. Res. 75, 4538-4547. https://doi.org/10.1158/0008-5472.CAN-15-2171-T (2015).

11. Chou, W. C. et al. B-Myb induces APOBEC3B expression leading to somatic mutation in multiple cancers. Sci. Rep. 7 , 44089. https://doi.org/10.1038/srep44089 (2017).

12. Matsumoto, T. et al. Protein kinase A inhibits tumor mutator APOBEC3B through phosphorylation. Sci. Rep. 9, 8307. https://doi. org/10.1038/s41598-019-44407-9 (2019).

13. Rouf Banday, A. et al. Targeting natural splicing plasticity of APOBEC3B restricts its expression and mutagenic activity. Commun. Biol. 4, 386. https://doi.org/10.1038/s42003-021-01844-5 (2021).

14. Periyasamy, M. et al. APOBEC3B-mediated cytidine deamination is required for estrogen receptor action in breast cancer. Cell Rep. 13, 108-121. https://doi.org/10.1016/j.celrep.2015.08.066 (2015).

15. Chen, Y. et al. DHX9 interacts with APOBEC3B and attenuates the anti-HBV effect of APOBEC3B. Emerg. Microbes Infect. 9, 366-377. https://doi.org/10.1080/22221751.2020.1725398 (2020).

16. Cheng, A. Z. et al. Epstein-Barr virus BORF2 inhibits cellular APOBEC3B to preserve viral genome integrity. Nat. Microbiol. 4, 78-88. https://doi.org/10.1038/s41564-018-0284-6 (2019).

17. Wang, D. et al. APOBEC3B interaction with PRC2 modulates microenvironment to promote HCC progression. Gut https://doi. org/10.1136/gutjnl-2018-317601 (2019).

18. Cortez, L. M. et al. APOBEC3A is a prominent cytidine deaminase in breast cancer. PLoS Genet. 15, e1008545. https://doi.org/10. 1371/journal.pgen.1008545 (2019).

19. Iwatani, Y., Takeuchi, H., Strebel, K. \& Levin, J. G. Biochemical activities of highly purified, catalytically active human APOBEC3G: correlation with antiviral effect. J. Virol. 80, 5992-6002. https://doi.org/10.1128/JVI.02680-05 (2006).

20. McDougall, W. M. \& Smith, H. C. Direct evidence that RNA inhibits APOBEC3G ssDNA cytidine deaminase activity. Biochem. Biophys. Res. Commun. 412, 612-617. https://doi.org/10.1016/j.bbrc.2011.08.009 (2011).

21. Soros, V. B., Yonemoto, W. \& Greene, W. C. Newly synthesized APOBEC3G is incorporated into HIV virions, inhibited by HIV RNA, and subsequently activated by RNase H. PLoS Pathog. 3, e15. https://doi.org/10.1371/journal.ppat.0030015 (2007).

22. McDougall, W. M., Okany, C. \& Smith, H. C. Deaminase activity on single-stranded DNA (ssDNA) occurs in vitro when APOBEC3G cytidine deaminase forms homotetramers and higher-order complexes. J. Biol. Chem. 286, 30655-30661. https:// doi.org/10.1074/jbc.M111.269506 (2011).

23. Xiao, X. et al. Structural determinants of APOBEC3B non-catalytic domain for molecular assembly and catalytic regulation. Nucleic Acids Res. 45, 7494-7506. https://doi.org/10.1093/nar/gkx362 (2017).

24. Mishra, N., Reddy, K. S., Timilsina, U., Gaur, D. \& Gaur, R. Human APOBEC3B interacts with the heterogenous nuclear ribonucleoprotein A3 in cancer cells. J. Cell. Biochem. 119, 6695-6703. https://doi.org/10.1002/jcb.26855 (2018).

25. Zhang, W. et al. Cytidine deaminase APOBEC3B interacts with heterogeneous nuclear ribonucleoprotein K and suppresses hepatitis B virus expression. Cell. Microbiol. 10, 112-121. https://doi.org/10.1111/j.1462-5822.2007.01020.x (2008).

26. Yamazaki, H. et al. APOBEC3B reporter myeloma cell lines identify DNA damage response pathways leading to APOBEC3B expression. PLoS ONE 15, e0223463. https://doi.org/10.1371/journal.pone.0223463 (2020).

27. Szklarczyk, D. et al. STRING v11: protein-protein association networks with increased coverage, supporting functional discovery in genome-wide experimental datasets. Nucleic Acids Res. 47, D607-D613. https://doi.org/10.1093/nar/gky1131 (2019).

28. Denegri, M. et al. Stress-induced nuclear bodies are sites of accumulation of pre-mRNA processing factors. Mol. Biol. Cell 12, 3502-3514. https://doi.org/10.1091/mbc.12.11.3502 (2001).

29. Marchesini, M. et al. ILF2 is a regulator of RNA splicing and DNA damage response in 1q21-amplified multiple myeloma. Cancer Cell 32, 88-100. https://doi.org/10.1016/j.ccell.2017.05.011 (2017).

30. Mondal, S., Begum, N. A., Hu, W. \& Honjo, T. Functional requirements of AID's higher order structures and their interaction with RNA-binding proteins. Proc. Natl. Acad. Sci. U.S.A. 113, E1545-1554. https://doi.org/10.1073/pnas.1601678113 (2016).

31. Hu, W., Begum, N. A., Mondal, S., Stanlie, A. \& Honjo, T. Identification of DNA cleavage- and recombination-specific hnRNP cofactors for activation-induced cytidine deaminase. Proc. Natl. Acad. Sci. U.S.A. 112, 5791-5796. https://doi.org/10.1073/pnas. 1506167112 (2015).

32. Nemec, P. et al. Gain of 1q21 is an unfavorable genetic prognostic factor for multiple myeloma patients treated with high-dose chemotherapy. Biol. Blood Marrow Transplant. J. Am. Soc. Blood Marrow Transplant. 16, 548-554. https://doi.org/10.1016/j.bbmt. 2009.11.025 (2010). 
33. Hanamura, I. et al. Frequent gain of chromosome band 1q21 in plasma-cell dyscrasias detected by fluorescence in situ hybridization: Incidence increases from MGUS to relapsed myeloma and is related to prognosis and disease progression following tandem stem-cell transplantation. Blood 108, 1724-1732. https://doi.org/10.1182/blood-2006-03-009910 (2006).

34. Wu, K. L. et al. Abnormalities of chromosome 1p/q are highly associated with chromosome 13/13q deletions and are an adverse prognostic factor for the outcome of high-dose chemotherapy in patients with multiple myeloma. Br. J. Haematol. 136, 615-623. https://doi.org/10.1111/j.1365-2141.2006.06481.x (2007).

35. Klein, U. et al. Chromosomal aberrations $+1 \mathrm{q} 21$ and del(17p13) predict survival in patients with recurrent multiple myeloma treated with lenalidomide and dexamethasone. Cancer 117, 2136-2144. https://doi.org/10.1002/cncr.25775 (2011).

36. An, G. et al. Cytogenetic and clinical marks for defining high-risk myeloma in the context of bortezomib treatment. Exp. Hematol. 43, 168-176. https://doi.org/10.1016/j.exphem.2014.11.004 (2015).

37. Treon, S. P. et al. Elevated soluble MUC1 levels and decreased anti-MUC1 antibody levels in patients with multiple myeloma. Blood 96, 3147-3153. https://doi.org/10.1182/blood.V96.9.3147 (2000).

38. Zhang, B., Gojo, I. \& Fenton, R. G. Myeloid cell factor-1 is a critical survival factor for multiple myeloma. Blood $99,1885-1893$. https://doi.org/10.1182/blood.v99.6.1885 (2002).

39. Inoue, J. et al. Overexpression of PDZK1 within the 1q12-q22 amplicon is likely to be associated with drug-resistance phenotype in multiple myeloma. Am. J. Pathol. 165, 71-81. https://doi.org/10.1016/s0002-9440(10)63276-2 (2004).

40. Stephens, O. W. et al. An intermediate-risk multiple myeloma subgroup is defined by sIL-6r: Levels synergistically increase with incidence of SNP rs2228145 and 1q21 amplification. Blood 119, 503-512. https://doi.org/10.1182/blood-2011-07-367052 (2012).

41. Mani, M. et al. BCL9 promotes tumor progression by conferring enhanced proliferative, metastatic, and angiogenic properties to cancer cells. Can. Res. 69, 7577-7586. https://doi.org/10.1158/0008-5472.CAN-09-0773 (2009).

42. Zhan, F. et al. CKS1B, overexpressed in aggressive disease, regulates multiple myeloma growth and survival through SKP2- and p27Kip1-dependent and -independent mechanisms. Blood 109, 4995-5001. https://doi.org/10.1182/blood-2006-07-038703 (2007).

43. Shaughnessy, J. D. Jr. et al. Pharmacogenomics of bortezomib test-dosing identifies hyperexpression of proteasome genes, especially PSMD4, as novel high-risk feature in myeloma treated with Total Therapy 3. Blood 118, 3512-3524. https://doi.org/10.1182/ blood-2010-12-328252 (2011).

44. Fischle, W. et al. Enzymatic activity associated with class II HDACs is dependent on a multiprotein complex containing HDAC3 and SMRT/N-CoR. Mol. Cell 9, 45-57. https://doi.org/10.1016/s1097-2765(01)00429-4 (2002).

45. da Huang, W., Sherman, B. T. \& Lempicki, R. A. Systematic and integrative analysis of large gene lists using DAVID bioinformatics resources. Nat. Protoc. 4, 44-57. https://doi.org/10.1038/nprot.2008.211 (2009).

46. da Huang, W., Sherman, B. T. \& Lempicki, R. A. Bioinformatics enrichment tools: Paths toward the comprehensive functional analysis of large gene lists. Nucleic Acids Res. 37, 1-13. https://doi.org/10.1093/nar/gkn923 (2009).

47. Kanehisa, M. \& Goto, S. KEGG: Kyoto encyclopedia of genes and genomes. Nucleic Acids Res. 28, 27-30. https://doi.org/10.1093/ nar/28.1.27 (2000).

48. Kanehisa, M. Toward understanding the origin and evolution of cellular organisms. Protein Sci. Publ. Protein Soc. 28, 1947-1951. https://doi.org/10.1002/pro.3715 (2019).

49. Kanehisa, M., Furumichi, M., Sato, Y., Ishiguro-Watanabe, M. \& Tanabe, M. KEGG: Integrating viruses and cellular organisms. Nucleic Acids Res. 49, D545-D551. https://doi.org/10.1093/nar/gkaa970 (2021).

50. Sawasaki, T., Ogasawara, T., Morishita, R. \& Endo, Y. A cell-free protein synthesis system for high-throughput proteomics. Proc. Natl. Acad. Sci. U.S.A. 99, 14652-14657. https://doi.org/10.1073/pnas.232580399 (2002)

51. Endo, Y. \& Sawasaki, T. High-throughput, genome-scale protein production method based on the wheat germ cell-free expression system. Biotechnol. Adv. 21, 695-713. https://doi.org/10.1016/s0734-9750(03)00105-8 (2003).

\section{Acknowledgements}

This work was partly supported by JSPS KAKENHI Grant Numbers JP16K08809, JP19K07591 to K.S., JP19H03502, JP18H03992 to A.T-K., by AMED under Grant Numbers JP20cm0106501 and JP21fk0410034 to A.T-K., and a research Grant from ONO PHARMACEUTICAL CO. LTD. We thank Anamaria Daniela Sarca for editorial assistance.

\section{Author contributions}

Y.K., K.S. and A.T.-K. conceived the study; Y.K. carried out experiments with help from Y.T., H.Y., R.N., Y.H., S.T., E.S., H.M., T.M., K.S; F.T., and R.M. provided vital reagents; S.I. analyzed the MS data; Y.K., K.S. and A.T.-K. wrote the paper. All the authors reviewed and approved the manuscript.

\section{Competing interests}

The authors declare no competing interests.

\section{Additional information}

Supplementary Information The online version contains supplementary material available at https://doi.org/ 10.1038/s41598-022-06226-3.

Correspondence and requests for materials should be addressed to K.S.

Reprints and permissions information is available at www.nature.com/reprints.

Publisher's note Springer Nature remains neutral with regard to jurisdictional claims in published maps and institutional affiliations.

Open Access This article is licensed under a Creative Commons Attribution 4.0 International License, which permits use, sharing, adaptation, distribution and reproduction in any medium or format, as long as you give appropriate credit to the original author(s) and the source, provide a link to the Creative Commons licence, and indicate if changes were made. The images or other third party material in this article are included in the article's Creative Commons licence, unless indicated otherwise in a credit line to the material. If material is not included in the article's Creative Commons licence and your intended use is not permitted by statutory regulation or exceeds the permitted use, you will need to obtain permission directly from the copyright holder. To view a copy of this licence, visit http://creativecommons.org/licenses/by/4.0/.

(c) The Author(s) 2022 